\title{
Appreciating, Measuring and Incentivising Discipline Diversity: Meaningful Indicators of Collaboration in Research
}

\author{
Helen Hasan \\ Australian Health Services Research Institute \\ University of Wollongong \\ hasan@uow.edu.au \\ Linda Dawson \\ Faculty of Social Science \\ University of Wollongong \\ lindad@uow.edu.au
}

\section{Abstract}

Inter-disciplinary collaborative research is generally believed to lead to innovative outcomes in areas that may be missed in research studies based in a single discipline. However, currently available research performance indicators, based on scholarly peer-reviewed publications and citations from a single discipline, do little to recognise the merits of collaborative and interdisciplinary research. This paper presents an empirical study of members of a research unit and their publication and grant profiles. From analysis of this data a set of profile categories emerged together with the relevant indicators which provide a framework from which a deeper understanding of how different research behaviours contribute to the differences in researchers' individual profiles. These profiles could be used to provide a richer environment for the evaluation of research performance, both in terms of outputs and potential funding opportunities, and indicators of 'good research' in inter-disciplinary projects.

Keywords: Collaboration; diversity: research performance; indicators; inter-disciplinary

\section{Introduction}

Although widely used and generally found beneficial or convenient, currently available research performance indicators, based on scholarly peer-reviewed publications and citations from the same in a single discipline, do little to recognise the merits of collaborative and interdisciplinary research. Several scholars have suggested a broader range of academic research KPIs than those that are already used, which are often restricted to publication counts in "quality" journals and citations to these publications (Takeda et al 2010). Goring et al (2014) suggest that scholarly performance evaluation should include research outputs other than publications, including educational outcomes, dataset creation, outreach products (eg blogs or social media), and the application of scientific results to policy or management activities, similar to altmetrics (Priem, 2011). Iivari (2008) recommends a combination of expert evaluation and bibliometric evaluation. In evaluating the influence of respected IS scholar Heinz Klein, Truex et al (2011) propose the "focus should be shifted from the venue of publication of the research to the uptake of the ideas contained in it, thus increasing the openness of the discourse, participation in the discourse, truthfulness, and reduction of the inequities in power distribution within academia" (Truex et al 2011 p 422). Li et al (2013) suggest that by using social capital indicators "a scholar can identify his or her structural position in the network and formulate a co-authorship strategy according to the future position where he or she wants to be." ... [however] ... "collaborating with too many different scholars might put a researcher at risk of being distrusted by prolific scholars and losing chances to coauthor with them." p 1528.

These proposed means of assessing research performance are valuable but introduce a subjective element that would be difficult to implement. They also do not directly address issues of disciplinary diversity. The study that is presented in this paper provides a pragmatic 
way of measuring the extent of collaboration and inter-disciplinarity of a scholars work. Although these measures in themselves say little about research outcomes, they do facilitate the creation of different researcher profiles that could determine on what grounds researchers with these different profiles could be assessed for relevant quality outcomes.

\subsection{Strength from diversity}

Studies indicate that diverse teams that consisting of heterogeneous participants have the ability to be more innovative and more creative than teams consisting of very homogeneous members.

$$
\text { Beekhuyzen J . Stein A. (2014) }
$$

The above assertion in the Call for Papers is widely accepted and describes the motivation for this paper. There is an abundance of evidence that diversity in team membership is beneficial although it is not a simple matter with many types of diversity and finding a correlation between diversity and performance can be a challenge (Crawford \& Hasan 2009, Liao 2011, Podestá et al 2013, Kearney and Gebert 2009, Rafols and Meyer 2009). In a study of teams playing weekly strategy games, Crawford and Hasan (2009) found that heterogeneous teams were able to perform complex activities better than homogeneous ones after an initial learning period of 2 or 3 weeks. They observed that the heterogeneous teams required a few games played together to learn cooperative team skills and effective ways of communicating. However, once team members appreciated the abilities of others, they could effectively leverage their diverse capabilities to perform more effectively overtaking the homogeneous teams whose performances did not greatly improve over time. Podestá et al 2013 argue that "a self-reflective process to identify and intervene on factors that foster or impede cooperative production of knowledge should be an essential component of integrated assessments involving scientists, practitioners and stakeholders." P 40.

Research that crosses disciplinary boundaries or falls between or outsidetraditional disciplines is generally believed to lead to innovative outcomes in areas that may be missed in single discipline research studies (Liao 2011). According to Liao (2011) diversity leads to better research quality which not only inspires scholars to continue their research, but also increases the possibility of higher research budgets from sponsors. It is therefore to be encouraged through appropriate incentives and rewards. However, recognition of collaboration and interdisciplinarity are rarely included in the key performance indications (KPI), on which researchers in academic institutions are assessed for promotion and funding, what is more, inter-disciplinary projects invariably involve collaborations between researchers from different disciplines. A consequence of this is that along with the potential benefits of interdisciplinary research, there are challenges of communicating across varying disciplinary content, language and paradigms leading to challenges in getting inter-disciplinary work published in respected, discipline-focussed journals. To many scholars, these costs are a disincentive to appreciating the benefits of working in diverse teams. This is important for both individual scholars and research managers as "current reward structures are problematic for scientists engaged in interdisciplinary research, particularly early career researchers, because academic culture tends to value only some research outputs, such as primary-authored publications." (Goring et al 2014, p39). The focus of this paper is on the extent, or lack of, appreciation, measurement and incentivising of discipline diversity in research teams.

We propose that a new approach to the assessment of research quality is needed that will expand the scope of research performance indicators beyond individual single-disciplinary research. As a first step in gaining recognition for collaborative, inter-disciplinary research we take a critical look at research performance indicators in the next section, and in following sections describe an empirical study into suitable indicators relevant to their strengths and specific attributes of all researchers. The results of this study suggest ways that these indicators can be used to profile researchers so that their outputs and contributions can be assessed appropriately. 


\section{Research Performance Indicators}

The reliance on currently available research performance indicators, based on scholarly peerreviewed publications and citations from the same in a single discipline, is concerning as according to Rafols and Meyer (2009), "interdisciplinary research is seen as more successful in achieving breakthroughs and relevant outcomes, be it in terms of innovation for economic growth or for social needs." p 263.

In order to gain appropriate recognition for those who take up the challenges of collaborative research (within or across disciplines) and inter-disciplinary research (by an individual or group), we investigate potential research performance indicators that could be used by researchers to create their collaboration and inter-disciplinary profile. Thus, where researchers engage in inter-disciplinary research projects in order to achieve specific breakthroughs and innovation, they can be recognised as taking up additional challenges and their output could be judged accordingly. In the business of research, managers are required to make decisions on the allocation of funds and resources; on the appointment and promotion of researchers; on the formation of research units; and on whom to reward and encourage. These decisions take into account the quality of previous research performance and future research potential. There is, therefore an imperative to have reliable and meaningful indicators of research quality and potential.

A traditional indicator of past performance is a researcher's scholarly publication record and its impact. The quality of this record can be measured by the quantity of published work weighted by journal rankings. Impact is usually determined by including citations to those publications using indicators, such as the h-index (Hirsch 2005) where someone with an index of $h$ has published $h$ papers, which have been cited by others at least $h$ times and, in some disciplines, published impact factors of the journals themselves which have been shown to be problematic when considering research quality (Lamp et al, 2007). Research potential is more difficult to determine but can be based on expert scoring of a project proposal, the stage of the researcher's career, the research environment or the strength of partnerships with others.

It is well accepted that there are limitations of using metrics to judge quality. Donovan (2011) notes that: "Metrics-only approaches employing economic data and science, technology and innovation indicators were found to be behind the times: best practice combines narratives with relevant qualitative and quantitative indicators to gauge broader social, environmental, cultural and economic public value. Limited consultation between policy-makers and the research evaluation community has led to a lack of policy-learning from international developments." Further limitations include, performance indicators based on scholarly publications do not cover all attributes of what is considered "good research" in terms of its value, productivity, national benefit and wider impact. As scholarly publications are not the only output of research projects, reliance on the impact associated with citations in the same set of scholarly publications restricts the judgement of quality to the community of scholars in the researcher's academic discipline. Even then there is no guarantee that such citations confirm the quality of the research. They may refer to the method or theory underpinning the study rather than the study itself. Indeed, a citation may be quite critical or damning of the work.

The peer-review process has an inherent conservative bias (Luukkonen 2012.) This impacts on both the researcher's publication record and on the scoring of project proposals by expert panels. This bias also mitigates against the recognition of breakthrough ideas or innovative research proposals, which, as stated above, are the likely outcomes of collaborative, interdisciplinary research. It may even discourage researchers from engaging in inter-disciplinary innovative projects with potential to achieve breakthroughs in new areas. 


\section{Indicators of Collaboration and Cross-Disciplinarity}

\subsection{Background}

In our investigation of potential indicators that could be used by researchers to create their collaborative and inter-disciplinary profile, a literature review outlining approaches to measuring interdisciplinary scientific research (Wagner et al, 2011) provided inspiration and encouragement. These authors found that "key among the broader aspects are (a) characterizing the concept of knowledge integration, and (b) recognizing that it can occur within a single mind or as the result of team dynamics. Output measures alone cannot adequately capture this process. Among the quantitative measures considered, bibliometrics (co-authorships, collaborations, references, citations and co-citations) are the most developed, but leave considerable gaps in understanding."

The investigation reported here takes advantage of existing data within a university database (ResDB) on publications and grant applications of the university's researchers. As part of this data set, each researcher nominates their own 4 digit Field of Research (FOR) Code from the list provided by the Australian Research Council (ARC). Researchers can nominate more than one code but must allocate the relative percentage of each. Publication outlets such as journals and conferences are also allocated one or more FOR codes. While most researchers publish in outlets with their own nominated FOR, we are interested in those that also publish in other FOR codes and/ or with co-authors from other FOR codes.

\subsection{The Investigation}

Thirty-five members of a research unit participated in an exercise to examine their own publication and grant records. The University's Research Data Management Unit (RDMU) were contacted and asked to provide data from ResDB for each researcher on the following:

\subsection{Collaboration}

1. Number of co-authors on papers/ co-investigators on grant
a. Total number co-authors/co-investigators
b. Average number of authors/ investigators per publication/grant application
c. Number of distinct co-authors/ co-investigators and how many times they have collaborated
d. Number of co-authors/ co-investigators internal/ external to Faculty
e. Number of co-authors/ co-investigators internal/ external to University

\subsection{Multidisciplinary}

2. Number and percentages of different FOR codes of publications / grants

3. Number and percentages of different FOR codes among co-authors/coinvestigators

4. Possibility of weighting these by quality (eg publication ranking, grant amount)

The RDMU extracted publication and grant records for the 35 members of the research unit. In addition to this raw publication data, the researchers provided aggregated data on publication records which they itemised as follows:

- $\quad$ Total Number of co-authors - is the total of all co-author collaborations that occurred on a publication for all the publications of the researcher. The researcher does not contribute towards the count. So in essence, for each publication, its Total Authors on Pub - 1. 
- Total Number of co-authored publications - is the number of publications where there was a co-author collaborating on it. It does not include those publications which have been singularly authored by the researcher.

- $\quad$ Average number of co-authors per publication - is Total Number of coauthored publications / Total Number of co-authors.

- Number of Distinct co-authors - is the number of authors who have collaborated with the researcher.

- $\quad$ Average Distinct co-author per publication - Total number of co-authored pub / Number of Distinct co-authors

- $\quad$ Total Number of University Authors - The University authors are determined using the affiliation tick placed by the library on the publication records in ResDB.

- $\quad$ Number of external authors - every author that does not have the affiliation tick next to their name on the publication record in ResDB is deemed to be external.

- Number of co-authors with different FOR Codes - thenumber of co-authors who have a different FOR code as compared to the researcher.

- Total number of different FOR Codes for co-authors - the number of FOR Codes which the co-authors have that are not the same as those of the researcher.

- $\quad$ Number of Different FOR-Codes of Publications and relative numbers of each

Looking at the raw data as lists of publications in spread sheets, participant researchers, noted there was some missing or incorrect entries but not to any great extent. The FOR codes allocation to publications was obviously heavily skewed by the Australian ERA process with many being allocated values in line with one of the researcher's nominated FOR codes giving a distorted tendency to within-discipline alignment. However, the participants agreed that the aggregated data was meaningful as an indication of how they would classify themselves as researchers.

Each researcher was given their own data and asked to report on which of these items were meaningful to them; if there were any other items that could be included; and what personal research profile was revealed by these indicators. A workshop was held with the participant researchers and the RDMU team to discuss the data, its potential meaning and implications.

\section{Findings}

The participants agreed that it was important to emphasise that the proposed indicators are not measures of quality research in themselves, i.e. high scores are not inherently better than low ones. Rather, they would contribute to a researcher's holistic profile, providing evidence for whether they work alone or with others and whether their work lies within or across disciplines. The participants were asked to use their own indicators to create profiles in order to support the story the researcher could tell of their research when, for example, they are applying for grants or promotion. More significantly, the profiles could also be used to reassess how the value of their research can be determined and, in particular, whether existing indicators of research performance are appropriate or whether others should be used.

In the Appendix are Tables of the aggregated publication data for 29 participants (6 participants with less than 10 publications were removed). Each row represents data from one researcher participant. Tables 1-3 contain the same data but are sorted on three relevant indicators. The first column contains the number of the most appropriate profile (1-6) for the researcher whose data is contained in that row [NB: some are still unclassified]

The following are the set of six profiles that emerged together with the relevant indicators and range of scoring on those indicators [in brackets] on which they would be based: 
1. A lone researcher in a single discipline [few/no co-authors or co-investigators, publishes in their own FOR code i.e. scores low on all Tables]

2. A lone inter-disciplinary researcher [few/no co-authors or co-investigators, publishes in many FOR codes i.e. scores low on Tables 1 and 2 but high on Tables 3 and 4]

3. A researcher who collaborates with the same set of co-authors within a single discipline [many, but few distinct, co-authors and co-investigators in the same FOR code, publishes in the common FOR code i.e. scores high on Table 1 but low on Tables 2, 3 and 4]

4. A researcher who collaborates with the same set of co-authors from other disciplines [many, but few distinct, co-authors and co-investigators with different FOR codes, publishes in different FOR codes i.e. scores high on Table 1, 3 and 4 but low on Table 2]

5. A researcher who collaborates with many different co-authors within a single discipline [many distinct co-authors and co-investigators with the same FOR code, publishes in this FOR codei.e. scores high on Tables 1 and 2 but low on Table 3 and 4],

6. A researcher who collaborates with many different co-authors across many disciplines [many distinct co-authors and co-investigators in many FOR codes, publishes in many FOR codes i.e. scores high on all Tables]

\section{Analysis}

Although the various scores are only indicative of how to allocate researchers to the six categories, all participants that have so far been categorised agreed that their profile made sense to them. From discussion with the participants, the six profiles are now designated as follows:

1. A lone researcher in a single discipline

2. A lone inter-disciplinary researcher

3. A researcher who works in a single discipline team

4. A researcher who works in an inter-disciplinary team.

5. A researcher who works with different researchers from their discipline

6. A researcher who works with different researchers from many disciplines

As a general trend we can say that existing research performance indicators are increasingly less appropriate as we move from Profile 1 to Profile 6.

The lone researchers (Profiles 1 and 2) were viewed as being those most appropriately evaluated by existing research performance indicators. They published in standard academic outlets although those in Profile 2 who were inter-disciplinary found it harder to get published.

Among those with a collaborative profile (Profiles 3-6) there was a clear distinction between those who collaborated with a regular team of co-authors and co-investigators (Profiles 3 and 4) and those who collaborated with a large number of different co-authors and co-investigators (Profiles 5 and 6). The former were more focussed and fitted the existing research performance culture well. The latter tended to supervise large numbers of research students and/ or were mentors to early career researchers. This meant that they had more publications in B and C ranked journal or conferences than the A and A* journals as would be expected of their status as in the Excellence in Research in Australia (ERA) ranking as published by the ARC in 2010. Note that rankings were removed from ERA in 2012.

Another aspect of the distinction between collaborating in a regular team (Profiles 3 and 4) and collaborating with many different other researchers, is expressed in the findings of 
research by Abbasi and Altmann (2011) who say that "scholars with strong ties (i.e., repeated co-authorships) show a better research performance than those with low ties (e.g., single coauthorships with many different scholars). The results related to efficiency show that scholars, who maintain a strong co-authorship relationship to only one co-author of a group of linked co-authors (i.e., co-authors that havejoint publications), perform better than those researchers with many relationships to the same group of linked co-authors".

An inherent problem with collaborative researchers is that, while their outputs come from a team, they are mostly judged as individuals for awards, appointments, promotion and even funding. This is often in competition with team members or other collaborators. Researchers are often required to allocate percentage contributions to all joint publications. Funding formulae based on publications are reduced by this contribution making collaboration less attractive.

Those with Profiles 2, 4 and 6 scoring highly on cross-disciplinary indicators have challenges getting their work published in outlets that are ranked highly on existing research performance indicators. These are predominantly embedded in the paradigm, methodology and language of a single discipline. This presents particular challenges for a lone inter-disciplinary researcher (Profile 2). Those with Profiles 4 and 6 have the advantage that journals in which they want to publish belong to the discipline of one research team. Indeed, the same basic paper can be legitimately published in more than one outlet in different disciplines. This raises the issue of the motivation for publication which can be both to reach an intended audience and to improve the researchers' publication records in line with existing research performance indicators.

The literature introduced earlier in the paper describes the particular benefits of collaborative interdisciplinary research in terms of innovation, new ideas and breakthroughs. These are often well ahead of accepted topics covered by conservative highly ranked journals. There may be a need to protect the innovation, new ideas and breakthroughs with patents but these are not always applicable or affordable. For most research it is enough to put the work in the public domain attributed to the researchers as authors and originators, and therefore owners of the idea, innovation or breakthrough. The researcher needs to ensure that these are not 'stolen' before they can be transformed into products that will reap some reward, and this has been known to happen during the peer-review process. For this reason, the work may be published first, somewhere other than in scholarly publications, often online, because of the need to publish quickly. There were cases among the participants who had published articles in outlets not ranked by the formal performance assessment process and these articles had been highly cited because they were ahead of the pack on a new idea or innovation.

\section{Implications}

The motivation for this paper is to give researchers some evidence for new ways in describing their research to others, stories that vary from "I am a researcher who works alone and publishes in a single discipline; the output of my research consists of publications in highlyranked scholarly journals so that its quality and values should bejudged by my h-index" to "I am a researcher who works with an inter-disciplinary team of collaborators, who supervised research students and mentors early career researchers the output of these research activities consists of publications in highly-ranked scholarly journals in several disciplines, reports of break-though ideas in online quick-release journals and papers in $\mathrm{B}$ and $\mathrm{C}$ journals and conferences co-authored with students and mentees".

As a result of this study the questions we pose for future research are:

- $\quad$ Do current measures (h-index etc.) ignore, hinder or promote innovations, new ideas and breakthroughs?

- What are indicators of 'good research' in inter-disciplinary projects - i.e. how do you measure progress towards innovation and breakthrough"? 
- $\quad$ How do we allow for the dichotomy that collaboration is inherently team- not individual based yet it is often individuals who are assessed?

\section{Conclusion}

This paper began with the general challenges of appreciating, measuring and incentivising diversity in order to reap the benefits of workforce diversity in organisations, particularly academic institutions. We have examined the particular case of appreciating, measuring and incentivising diversity in research teams looking at ways that individual researchers may be prepared to put in the extra efforts needed when working in multi-disciplinary teams in order to produce innovative outcomes in areas where this is greatly needed. Ultimately, expanded measures incorporating a wider range of indicators should better reflect theimportant work of both disciplinary and interdisciplinary teams at all career stages; assist evaluators of research to understand wider dimensions and impacts of research; and help sustain and stimulate a collaborative culture of inter-disciplinary research.

We assume that this will be of interest to conference participants who are themselves academic researchers. We suggest also that the results of this investigation may be relevant to nonacademic contexts where further research can be undertaken.

\section{References}

Abbasi A., Altmann J . 2011. "On the Correlation between Research Performance and Social Network Analysis Measures Applied to Research Collaboration Networks", Proceeding of HICSS 2011

Beekhuyzen, J. Stein, A. 2014. "Discovering the Potential of Demographic and Cultural Diversity" Track Description ACIS2014

Crawford, K. Hasan, H. M. (2009). A gaming system experience for work in heterogeneous, self-directed teams. Proceedings of Simtect Adelaide.

Donovan, C. 2011. "State of the art in assessing research impact: introduction to a special issue," Research Evaluation (20:3), September, pp 175- 179.

Goring, S., Weathers, K., Dodd W., Soranno P., Sweets L., Cheruvelil K., J on S Kominoski J ., RüeggJ ., Thorn A., Utz R. 2014 "Improving the culture of interdisciplinary collaboration in ecology by expanding measures of success," Frontier Ecological Environments (12:1), pp 39-47.

Hirsch, J . 2005. “An index to quantify an individual's scientific research output," Proceedings of the National Academy of Sciences, 102/46, pp 16569-16572.

Iivari, J. 2008. "Expert evaluation vs bibliometric evaluation: experiences from Finland," European J ournal of Information Systems 17, pp 169- 173.

Kearney, E., Gebert, D. 2009 “Managing diversity and enhancing team outcomes: The promise of transformational leadership," J ournal of Applied Psychology, (94:1), pp 77-89.

Li, E.Y., Liao, C.H., and Yen, H.R. 2013. "Co-Authorship Networks and Research Impact: A Social Capital Perspective," Research Policy (42:9), 11// , pp. 1515-1530.

Liao, C. 2011. "How to improve research quality? Examining the impacts of collaboration intensity and member diversity in collaboration networks," Scientometrics 86, pp 747761.

Luukkonen, T. 2012. "Conservatism and risk-taking in peer review: Emerging ERC practices", Research Evaluation, 21, pp 48-60

Lamp, J., Milton, S., Dawson, L. and Fisher, J. 2007. "RQF Publication Quality Measures: Methodological Issues", in Proceedings of 18th Australasian Conference on Information Systems, Toowoomba, Australia. 
Podestá, G.P., Natenzon, C.E., Hidalgo, C., and Ruiz Toranzo, F. 2013. “Interdisciplinary Production of Knowledge with Participation of Stakeholders: A Case Study of a Collaborative Project on Climate Variability, Human Decisions and Agricultural Ecosystems in the Argentine Pampas," Environmental Science \& Policy (26:0), 2// , pp. 40-48.

Priem, J. 2011. "altmetrics: a manifesto", URL: http://altmetrics.org/manifesto/, retrieved October 2014.

Rafols I., Meyer M 2009. "Diversity and network coherence as indicators of interdisciplinarity: Case studies in bionanoscience," Scientometrics (82:2) pp 263-287.

TakedaH., Truex D., Cuellar M. 2010. "Evaluating Scholarly Influence Through Social Network Analysis: the Next Step in Evaluating Scholarly Influence," AMCIS 2010 Proceedings. Paper 573.

Truex D., Cuellar M., Takeda H., Vidgen R. 2011. "The scholarly influence of Heinz Klein: ideational and social measures of his impact on IS research and IS scholars," European J ournal of Information Systems 20, pp 422-439.

Wagner, C. S., J . D. Roessner, K. Bobb, J . T. Klein, K. W. Boyack, J . Keyton, I. Rafols and K. Börner 2011. "Approaches to understanding and measuring interdisciplinary scientific research (IDR): A review of the literature." J ournal of Informetrics 5(1): 14-26.

\section{Acknowledgements}

Substantial contributions to the research behind this paper were made by members of the THEORI Research group in the Faculty of Business at the University of Wollongong. The authors particularly acknowledge the work of Drs Kate Crawford, Alanah Kazlauskas and Carole Alcock. 


\section{Appendix}

\begin{tabular}{|c|c|c|c|c|c|c|c|c|}
\hline Profile & $\begin{array}{l}\text { Total co- } \\
\text { authors } \\
\text { on Pub }\end{array}$ & $\begin{array}{l}\text { Total co- } \\
\text { authored } \\
\text { Pubs }\end{array}$ & $\begin{array}{c}\text { Avg co- } \\
\text { authors / } \\
\text { Pub }\end{array}$ & $\begin{array}{l}\text { Num } \\
\text { distinct } \\
\text { co- } \\
\text { authors }\end{array}$ & $\begin{array}{l}\text { Avg distinct } \\
\text { co-authors } \\
\text { / pub }\end{array}$ & $\begin{array}{l}\text { Num of } \\
\text { Authors } \\
\text { Other } \\
\text { FOR } \\
\text { Codes } \\
\end{array}$ & $\begin{array}{c}\text { Number } \\
\text { of Other } \\
\text { FOR } \\
\text { Codes }\end{array}$ & $\begin{array}{c}\text { Spread of } \\
\text { FOR codes } \\
\text { in pubs }\end{array}$ \\
\hline 6 & 19 & 6 & 3.17 & 12 & 2 & 7 & 5 & 5 \\
\hline 5 & 11 & 4 & 2.75 & 11 & 2.75 & 2 & 2 & 2 \\
\hline 4 & 170 & 68 & 2.5 & 33 & 0.49 & 6 & 3 & 6 \\
\hline 6 & 101 & 42 & 2.4 & 49 & 1.17 & 3 & 3 & 5 \\
\hline \multirow[t]{4}{*}{4} & 85 & 37 & 2.3 & 19 & 0.51 & 4 & 3 & 7 \\
\hline & 131 & 59 & 2.22 & 45 & 0.76 & 8 & 6 & 3 \\
\hline & 21 & 10 & 2.1 & 10 & 1 & 1 & 2 & 7 \\
\hline & 27 & 13 & 2.08 & 20 & 1.54 & 15 & 7 & 1 \\
\hline 5 & 55 & 28 & 1.96 & 21 & 0.75 & 0 & 0 & 1 \\
\hline 3 & 19 & 10 & 1.9 & 3 & 0.3 & 0 & 0 & 1 \\
\hline 5 & 58 & 31 & 1.87 & 32 & 1.03 & 0 & 0 & 1 \\
\hline 3 & 26 & 14 & 1.86 & 14 & 1 & 1 & 2 & 1 \\
\hline 6 & 257 & 142 & 1.81 & 107 & 0.75 & 25 & 14 & 9 \\
\hline 5 & 163 & 92 & 1.77 & 61 & 0.66 & 12 & 9 & 4 \\
\hline 6 & 45 & 26 & 1.73 & 21 & 0.81 & 11 & 8 & 4 \\
\hline 6 & 50 & 29 & 1.72 & 27 & 0.93 & 10 & 8 & 5 \\
\hline 5 & 10 & 6 & 1.67 & 5 & 0.83 & 0 & 0 & 1 \\
\hline 5 & 39 & 24 & 1.63 & 16 & 0.67 & 1 & 1 & 1 \\
\hline 6 & 75 & 47 & 1.6 & 33 & 0.7 & 7 & 7 & 7 \\
\hline 3 & 41 & 26 & 1.58 & 16 & 0.62 & 0 & 0 & 1 \\
\hline \multirow[t]{2}{*}{6} & 32 & 21 & 1.52 & 17 & 0.81 & 1 & 2 & 3 \\
\hline & 82 & 56 & 1.46 & 40 & 0.71 & 4 & 3 & 0 \\
\hline \multirow[t]{2}{*}{5} & 31 & 23 & 1.35 & 18 & 0.78 & 2 & 1 & 4 \\
\hline & 64 & 48 & 1.33 & 29 & 0.6 & 3 & 2 & 7 \\
\hline \multirow[t]{2}{*}{2} & 41 & 31 & 1.32 & 13 & 0.42 & 5 & 10 & 6 \\
\hline & 39 & 31 & 1.26 & 23 & 0.74 & 3 & 4 & 3 \\
\hline 1 & 11 & 10 & 1.1 & 4 & 0.4 & 1 & 2 & 2 \\
\hline 1 & 20 & 19 & 1.05 & 7 & 0.37 & 0 & 0 & 0 \\
\hline 2 & 24 & 23 & 1.04 & 10 & 0.43 & 1 & 2 & 6 \\
\hline
\end{tabular}

Table 1 Publication data ordered by Avg co-authors/ Publication 


\begin{tabular}{|c|c|c|c|c|c|c|c|c|}
\hline Profile & $\begin{array}{c}\text { Total } \\
\text { Co- } \\
\text { authors } \\
\text { on Pub }\end{array}$ & $\begin{array}{l}\text { Total co- } \\
\text { authored } \\
\text { Pubs }\end{array}$ & $\begin{array}{c}\text { Avg co- } \\
\text { authors/Pub }\end{array}$ & $\begin{array}{l}\text { Num } \\
\text { distinct } \\
\text { co- } \\
\text { authors }\end{array}$ & $\begin{array}{l}\text { Avg distinct } \\
\text { co- } \\
\text { authors/pub }\end{array}$ & $\begin{array}{c}\text { Num of } \\
\text { Authors } \\
\text { Other } \\
\text { FOR } \\
\text { Codes }\end{array}$ & $\begin{array}{c}\text { Number } \\
\text { of Other } \\
\text { FOR } \\
\text { Codes }\end{array}$ & $\begin{array}{l}\text { Spread } \\
\text { of FOR } \\
\text { codes } \\
\text { in pubs }\end{array}$ \\
\hline 5 & 11 & 4 & 2.75 & 11 & 2.75 & 2 & 2 & 2 \\
\hline \multirow[t]{2}{*}{6} & 19 & 6 & 3.17 & 12 & 2 & 7 & 5 & 5 \\
\hline & 27 & 13 & 2.08 & 20 & 1.54 & 15 & 7 & 1 \\
\hline 6 & 101 & 42 & 2.4 & 49 & 1.17 & 3 & 3 & 5 \\
\hline \multirow[t]{2}{*}{5} & 58 & 31 & 1.87 & 32 & 1.03 & 0 & 0 & 1 \\
\hline & 21 & 10 & 2.1 & 10 & 1 & 1 & 2 & 7 \\
\hline 3 & 26 & 14 & 1.86 & 14 & 1 & 1 & 2 & 1 \\
\hline 6 & 50 & 29 & 1.72 & 27 & 0.93 & 10 & 8 & 5 \\
\hline 5 & 10 & 6 & 1.67 & 5 & 0.83 & 0 & 0 & 1 \\
\hline 6 & 45 & 26 & 1.73 & 21 & 0.81 & 11 & 8 & 4 \\
\hline 6 & 32 & 21 & 1.52 & 17 & $\mathbf{0 . 8 1}$ & 1 & 2 & 3 \\
\hline \multirow[t]{2}{*}{5} & 31 & 23 & 1.35 & 18 & 0.78 & 2 & 1 & 4 \\
\hline & 131 & 59 & 2.22 & 45 & 0.76 & 8 & 6 & 3 \\
\hline 5 & 55 & 28 & 1.96 & 21 & 0.75 & 0 & 0 & 1 \\
\hline \multirow[t]{3}{*}{6} & 257 & 142 & 1.81 & 107 & 0.75 & 25 & 14 & 9 \\
\hline & 39 & 31 & 1.26 & 23 & 0.74 & 3 & 4 & 3 \\
\hline & 82 & 56 & 1.46 & 40 & 0.71 & 4 & 3 & 0 \\
\hline 6 & 75 & 47 & 1.6 & 33 & 0.7 & 7 & 7 & 7 \\
\hline 5 & 39 & 24 & 1.63 & 16 & 0.67 & 1 & 1 & 1 \\
\hline 5 & 163 & 92 & 1.77 & 61 & 0.66 & 12 & 9 & 4 \\
\hline \multirow[t]{2}{*}{3} & 41 & 26 & 1.58 & 16 & 0.62 & 0 & 0 & 1 \\
\hline & 64 & 48 & 1.33 & 29 & 0.6 & 3 & 2 & 7 \\
\hline 4 & 85 & 37 & 2.3 & 19 & 0.51 & 4 & 3 & 7 \\
\hline 4 & 170 & 68 & 2.5 & 33 & 0.49 & 6 & 3 & 6 \\
\hline 2 & 24 & 23 & 1.04 & 10 & 0.43 & 1 & 2 & 6 \\
\hline 2 & 41 & 31 & 1.32 & 13 & 0.42 & 5 & 10 & 6 \\
\hline 1 & 11 & 10 & 1.1 & 4 & 0.4 & 1 & 2 & 2 \\
\hline 1 & 20 & 19 & 1.05 & 7 & 0.37 & 0 & 0 & 0 \\
\hline 3 & 19 & 10 & 1.9 & 3 & 0.3 & 0 & 0 & 1 \\
\hline
\end{tabular}

Table 2 Publication data ordered by Avg distinct co-authors/ publication 


\begin{tabular}{|c|c|c|c|c|c|c|c|c|}
\hline Profile & $\begin{array}{c}\text { Total } \\
\text { co- } \\
\text { authors } \\
\text { on Pub }\end{array}$ & $\begin{array}{l}\text { Total co- } \\
\text { authore } \\
\text { d Pubs }\end{array}$ & $\begin{array}{l}\text { Avgco- } \\
\text { authors / } \\
\text { Pub }\end{array}$ & $\begin{array}{l}\text { Num } \\
\text { distinct } \\
\text { co- } \\
\text { authors }\end{array}$ & $\begin{array}{c}\text { Avg } \\
\text { distinct } \\
\text { co-authors } \\
\text { / pub }\end{array}$ & $\begin{array}{c}\text { Num of } \\
\text { Author } \\
\text { s Other } \\
\text { FOR } \\
\text { Code }\end{array}$ & $\begin{array}{c}\text { Numbe } \\
\text { r of } \\
\text { Other } \\
\text { FOR } \\
\text { Codes }\end{array}$ & $\begin{array}{c}\text { Spread } \\
\text { of FOR } \\
\text { codes } \\
\text { in pubs }\end{array}$ \\
\hline \multirow[t]{2}{*}{6} & 257 & 142 & 1.81 & 107 & 0.75 & 25 & 14 & 9 \\
\hline & 27 & 13 & 2.08 & 20 & 1.54 & 15 & 7 & 1 \\
\hline 5 & 163 & 92 & 1.77 & 61 & 0.66 & 12 & 9 & 4 \\
\hline 6 & 45 & 26 & 1.73 & 21 & 0.81 & 11 & 8 & 4 \\
\hline \multirow[t]{2}{*}{6} & 50 & 29 & 1.72 & 27 & 0.93 & 10 & 8 & 5 \\
\hline & 131 & 59 & 2.22 & 45 & 0.76 & 8 & 6 & 3 \\
\hline 6 & 75 & 47 & 1.6 & 33 & 0.7 & 7 & 7 & 7 \\
\hline 6 & 19 & 6 & 3.17 & 12 & 2 & 7 & 5 & 5 \\
\hline 4 & 170 & 68 & 2.5 & 33 & 0.49 & 6 & 3 & 6 \\
\hline \multirow[t]{2}{*}{2} & 41 & 31 & 1.32 & 13 & 0.42 & 5 & 10 & 6 \\
\hline & 82 & 56 & 1.46 & 40 & 0.71 & 4 & 3 & 0 \\
\hline 4 & 85 & 37 & 2.3 & 19 & 0.51 & 4 & 3 & 7 \\
\hline \multirow[t]{3}{*}{6} & 39 & 31 & 1.26 & 23 & 0.74 & 3 & 4 & 3 \\
\hline & 101 & 42 & 2.4 & 49 & 1.17 & 3 & 3 & 5 \\
\hline & 64 & 48 & 1.33 & 29 & 0.6 & 3 & 2 & 7 \\
\hline 5 & 11 & 4 & 2.75 & 11 & 2.75 & 2 & 2 & 2 \\
\hline \multirow[t]{2}{*}{5} & 31 & 23 & 1.35 & 18 & 0.78 & 2 & 1 & 4 \\
\hline & 21 & 10 & 2.1 & 10 & 1 & 1 & 2 & 7 \\
\hline 3 & 26 & 14 & 1.86 & 14 & 1 & 1 & 2 & 1 \\
\hline 6 & 32 & 21 & 1.52 & 17 & 0.81 & 1 & 2 & 3 \\
\hline 5 & 24 & 23 & 1.04 & 10 & 0.43 & 1 & 2 & 6 \\
\hline 2 & 11 & 10 & 1.1 & 4 & 0.4 & 1 & 2 & 2 \\
\hline 1 & 39 & 24 & 1.63 & 16 & 0.67 & 1 & 1 & 1 \\
\hline 5 & 58 & 31 & 1.87 & 32 & 1.03 & $\mathbf{0}$ & 0 & 1 \\
\hline 5 & 10 & 6 & 1.67 & 5 & 0.83 & $\mathbf{0}$ & 0 & 1 \\
\hline 5 & 55 & 28 & 1.96 & 21 & 0.75 & $\mathbf{0}$ & 0 & 1 \\
\hline 3 & 41 & 26 & 1.58 & 16 & 0.62 & $\mathbf{0}$ & 0 & 1 \\
\hline 1 & 20 & 19 & 1.05 & 7 & 0.37 & $\mathbf{0}$ & 0 & 0 \\
\hline 3 & 19 & 10 & 1.9 & 3 & 0.3 & $\mathbf{0}$ & 0 & 1 \\
\hline
\end{tabular}

Table 3 Publication data ordered by Num of co-Authors Other FOR Code

An earlier version of this paper was presented at the Australasian Conference on Information Systems (ACIS) 2014 in Auckland, New Zealand.

Copyright: (c) 2015 Hasan \& Dawson. This is an open-access article distributed under the terms of the Creative Commons Attribution-NonCommercial 3.0 Australia License, which permits non-commercial use, distribution, and reproduction in any medium, provided the original author and AJ IS are credited. 


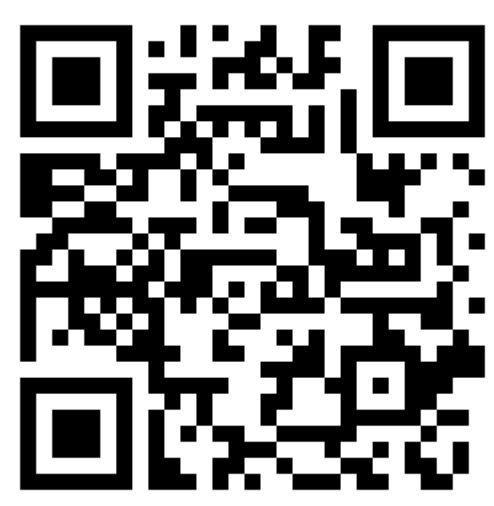

